\title{
HIGH-REDSHIFT RADIO GALAXIES
}

\author{
GEORGE MILEY \\ Leiden Observatory, Postbus 9513, 2300 RA Leiden, \\ The Netherlands
}

Radio galaxies are unique cosmological probes. As with radio-loud quasars, the presence of luminous radio continuum and optical line emission enable radio galaxies to be observed and recognized at large distances, up to $z=$ 4.2. However, unlike the situation for most quasars, their optical emission can be spatially resolved from the ground and studied in detail.

Progress in detecting distant radio galaxies has been rapid in recent years due to the use of CCDs and the exploitation of new selection criteria. Now, more than 60 radio galaxies are known with $z>2$. More than half of these have been found by our group by concentrating on radio sources with the steepest spectra, most of these in a "Key Programme" of the European Southern Observatory. Although several people contributed to this Key Programme, most of the work was done by Huub Röttgering, who presented his Ph.D thesis in January and Rob van Ojik, who succeeded him. Redshifts of 1.5 to 4 correspond to a time when the Universe was $10 \%-20 \%$ of its present age. This was a crucial period in history when galaxy formation must have been rampant. It corresponds to the AGN era, a two-billion year "delta function" in the population evolution of luminous quasars and radio galaxies, when their space-density rose to a value several hundred times larger than the present density before the species mysteriously and suddenly became almost extinct.

Radio galaxies are extremely valuable for studying this period of the Universe because they emit three components, (IR-optical-UV continuum, emission lines and radio continuum) that are all highly luminous and spatially extended. Not only can different and complementary sets of diagnostics be derived from each of these components, but studies of the relationships between these various diagnostics and the interaction between the different components provides yet additional knowledge about the physical conditions. Such data are relevant for understanding the formation and evolution of galaxies, active nuclei and radio sources. 
The identified objects have R-magnitudes which are typically between 21.5 and 24 . One of the most intriguing properties of distant radio galaxies is that their UV/optical/IR continua are preferentially aligned along the radio sources (e.g. Chambers et al. 1987). HST observations have shown that the alignment can occur even on the sub-kiloparsec scale and that the emission is highly clumped (Miley et al. 1992, Miley 1993). Several classes of models have been proposed to account for the alignment effect (McCarthy 1993). Starburst models, interpret the alignment as due to star formation stimulated by the radio jet as it propagates outward from the nucleus (e.g. Rees 1989; De Young 1989; Begelman and Cioff 1989). Another possibility is that the aligned emission is indirect light from a hidden quasar scattered by electrons (Fabian 1989) or dust (Tadhunter et al. 1989). The scattering model was stimulated by the discovery that the extended emission is sometimes polarized in the UV.

Some evidence for starbursting comes from recent work by van Ojik et al. (1994). We show that the spectrum of TX0211-122, a $z=2.3$ galaxy discovered during the ESO Key Programme is highly anomalous with a flux ratio of $\operatorname{Ly} \alpha$ to NV $\lambda 1240$ which is a factor of 30 smaller than for a typical highredshift radio galaxy. The is unlike that of any other extragalactic object except that of the $z=2.3$ IRAS galaxy F10214+4724, which is also a substantial radio source. In both objects depression of Ly $\alpha$ appears to be accompanied by simultaneous enhancement of NV. The relatively large NV/CIV ratio implies that the lineemitting gas is overabundant in nitrogen. The most feasible explanation for the spectra is that both objects have undergone a period of intense star formation. The most massive stars would evolve quickly, producing (i) the dust responsible for attenuating the Ly $\alpha$ and (ii) a relative overabundance of nitrogen.

Most of the evidence for alignment of the UV/optical/IR continuum with the radio emission is based on broad-band images. IR spectroscopy by Eales and Rawlings (1993) indicate that a substantial part of the broad-band $\mathrm{K}$ flux is affected by the presence of emission by $\mathrm{H} \alpha$ or [OIII] in the band. The strong lines are indeed a serious contaminant in the IR, but for $\mathrm{z}>1.2$, they have a negligible effect in the $\mathrm{R}$-band images. The radio/IR alignment effect is more suspect. However, a beautiful recent image of $4 \mathrm{C} 41.17$ at $\mathrm{z}$ $=3.8$ taken by the Keck telescope in $K^{\prime}$ band excludes emission lines, but shows that the light emitted near $5000 \AA$ is clearly aligned along the radio axis (Graham et al. 1993).

Recent observations pose difficulties for all the various models proposed to explain the alignment effect and it is unlikely that any one of the proposed explanations, by itself, can account for the UV/optical/IR continuum. In my view a combination of jet-induced star formation together with one or both of the scattering hypotheses are likely to be jointly responsible. 
One of the most interesting recent results is that a large number of highredshift radio galaxies appear to be double, with the axis of the optical double preferentially oriented along the radio axes. A statistical analysis of this effect was made by Röttgering (1993), who considered 31 high-redshift radio galaxies with radio sizes of between 4" and 20 ". He found that the tendency of "companions" to lie close to the radio axes is significant at the $5 \%$ level. One possible explanation is that the radio galaxies are undergoing vigorous merging with companions. Suppose that mergers were common during the $\mathrm{z}=2$ epoch and that either the starburst or scattering mechanism for enhancing luminosity occurs. When the merging galaxies are located along the beam, their luminosity would be enhanced compared with other merging galaxies and their fluxes become comparable with the parent radio galaxies.

Some evidence that the highest redshift galaxies are indeed surrounded by a cluster or group of less luminous galaxies is provided by the Keck picture of 4C 41.17 (Graham et al. 1993). A speculative person might conjecture on this basis that high-redshift radio galaxies are the progenitors of $\mathrm{cD}$ galaxies at the centres of still-forming rich clusters.

For the last 25 years one of the most important methods for investigating the early Universe has been to study quasar absorption lines. There are two limitations of such studies due to the fact that the light being absorbed originates in quasars. First, the quasars are unresolved, so in general no information can be obtained about the spatial scale of the absorbers. Secondly, the absorption lines having $z_{\mathrm{em}} / \operatorname{sim}$ to $\mathrm{z}_{\mathrm{abs}}$, i.e. those close to the quasar, are attenuated (presumably ionized) due to their proximity to the QSO. Absorption studies of radio galaxies would be hampered by neither of these effects. We (Hunstead, Röttgering, Miley, van Ojik and Wieringa) therefore recently instigated a project to search for absorption lines in high-redshift radio galaxies. Our initial targets were selected from galaxies having the brightest Ly $\alpha$. It is in the region of this strong line that we would have the most chance of seeing absorption. Preliminary results indicate that absorption features in the Ly $\alpha$ profiles are common. In one object, 0943-242, we see a sharp deep absorption trough that reaches zero intensity and has a half-width of more than $400 \mathrm{~km} / \mathrm{s}$. The probability of obtaining such a system by a chance on the basis of the statistics of quasar absorption lines is less than 1 in 1000 . The absorbers must therefore be intimately connected with the galaxy itself, or the environment of the galaxy, e.g. a protocluster. The depth of the absorption trough implies that the absorbing cloud at least covers the complete Ly $\alpha$ emission region (> $30 \mathrm{kpc}$ ). Although the exact column density involved is dependant on the 
number of absorbing clouds involved, the value will lie between $10^{18}$ and $10^{20} \mathrm{~cm}^{-2}$, implying a total mass in $\mathrm{HI}$ of approximately $10^{7} \mathrm{M}_{0}$. There appears to be a velocity shift along the slit of roughly $10 \mathrm{~km} / \mathrm{s}$ which, if interpreted as rotation would imply a dynamic mass of $10^{9} \mathrm{M}_{0}$. A radio jet impacting on an object of such a mass could easily be deflected (van Breugel et al. 1985). It is tempting to speculate that the culprit responsible for the Ly $\alpha$ absorption is similar to one of the companion objects visible in the Keck picture of $4 \mathrm{C} 41.17$.

I draw the following conclusions with various degrees of certainty:

(i) High-redshift radio galaxies are the progenitors of giant elliptical galaxies, located near the centres of protoclusters, maybe at the centres of giant cooling flows.

(ii) They are undergoing vigorous star formation which is producing dust.

(iii) The radio/optical alignments are produced by a combination of jetinduced star formation and scattering of light from a hidden quasar, in the parent galaxy and in nearby companions.

(iv) The galaxies are located in highly inhomogeneous regions of the $z=2$ Universe.

High-redshift radio galaxies will provide one of the most important tools for studying the early Universe, using the present and next generations of large telescopes. Their scales are particularly well-suited to study with the HST. Selecting objects with extreme radio spectra from the large-sky surveys at present underway with the Westerbork, VLA and Molonglo telescope will provide a rich harvest for many decades in the future.

I thank my collaborators, particularly Huub Röttgering, Richard Hunstead and Rob van Ojik.

\section{References}

Begelman, M.C., and Cioffi, D.F., (1989), Ap.J. (Letters), Vol. 345, pp. L21

van Breugel, W.J.M., Miley, G. K.,Heckman, T., Butcher, H. and Bridle, A., (1985), Ap. J. Vol. 290 , pp. 496

Chambers, Miley and van Breugel, (1987), Nature, Vol. 329, pp. 604

Chambers, K.C. Miley, G.K. and van Breugel, W.J.M., (1990), Ap. J., Vol. 363, pp. 21

De Young, D. S., (1989), Ap. J. (Letters), Vol. 342, pp. L59

Eales, S. A. and Rawlings, S., (1993), Ap. J., Vol. 411, pp. 67

Fabian, (1989), M.N.R.A.S., Vol. 243, pp. 1

Graham, J.,et al., (1993), Ap.J. (Letters), Vol. 420, pp. L5

McCarthy, P.J., (1993), Ann. Rev. Astron. Astrophys., Vol. 31, pp. 639

Rees, M.J., (1989), M.N.R.A.S., Vol. 239, pp. 1

Röttgering, H., (1993), Ph.D Thesis, University of Leiden

Röttgering, H., Miley, G.K., van Ojik, R., (1994), in preparation

Tadhunter, C.N., Fosbury, R.A.E., and di Sergio Aligheri, S., (1989), "Proc. of the Como

Conference on BL Lac Objects : 10 Years After", ed. L. Marasci 